Research Paper

\title{
Does the Preoperative Prognostic Nutritional Index Predict Survival in Patients with Liver Metastases from Colorectal Cancer Who Underwent Curative Resection?
}

\author{
Yujie Zhao"\#, Yuxiang Deng"\#, Jianhong Peng' ${ }^{1}$, Qiaoqi Sui ${ }^{1}$, Junzhong Lin ${ }^{1}$, Miaozhen Qiu $^{2}$, Zhizhong \\ $\operatorname{Pan}^{1 凶}$ \\ 1. Department of Colorectal Surgery, State Key Laboratory of Oncology in South China, Collaborative Innovation Center for Cancer Medicine, Sun Yat-sen \\ University Cancer Center, Guangzhou, China. \\ 2. Department of Medical Oncology, State Key Laboratory of Oncology in South China, Sun Yat-sen University Cancer Center, Guangzhou, P.R. China. \\ \#Both authors equally contributed to this manuscript
}

$\square$ Corresponding author: Zhizhong Pan, Department of Colorectal Surgery, State Key Laboratory of Oncology in South China, Collaborative Innovation Center for Cancer Medicine, Sun Yat-sen University Cancer Center, 651 Dongfeng East Road, Guangzhou 510060, P.R.China. Phone:+86-20-87343124; Fax: +86-20-87343609; E-mail: panzhzh@sysucc.org.cn

( $)$ Ivyspring International Publisher. This is an open access article distributed under the terms of the Creative Commons Attribution (CC BY-NC) license (https://creativecommons.org/licenses/by-nc/4.0/). See http://ivyspring.com/terms for full terms and conditions.

Received: 2018.02.04; Accepted: 2018.04.13; Published: 2018.05.25

\begin{abstract}
Purpose: The prognostic nutritional index (PNI) has been correlated with long-term outcomes in various cancer patients. However, the relationship between the PNI and long-term outcomes in patients with colorectal cancer liver metastasis (CRLM) who have undergone liver surgery have not been fully investigated. In this study, we aimed to identify the impact of the preoperative $\mathrm{PNI}$ on the long-term oncologic outcomes of patients with CRLM who have undergone curative hepatic resection.

Methods: A total of 243 CRLM patients who underwent curative hepatic resection for liver metastases in the Sun Yat-sen University Cancer Center between September 1999 and July 2015 were enrolled, and their medical records were analyzed retrospectively. The preoperative PNI was calculated as $10 \times$ the serum albumin concentration $(\mathrm{g} / \mathrm{dL})+0.005 \times$ the total lymphocyte count (per $\mathrm{mm}^{3}$ ). The PNI was compared according to the statuses of clinicopathological features. In addition, the regression-free survival (RFS) and overall survival (OS) were analyzed according to the preoperative $\mathrm{PNI}$ using univariate and multivariate analyses.

Results: The optimal cut-off value of the preoperative PNI was set at 48.5 using the X-tile software. Older patients and those who had undergone synchronous hepatic resection were more likely to belong to the low PNI group ( $\leq 48.5$ ) (all $\mathrm{P}<0.05$ ). In multivariate analyses, PNI $>48.5$ was associated with markedly better survival outcomes as an independent factor, both for OS and RFS.

Conclusion: For patients with CRLM undergoing curative hepatic resection, preoperative PNI is a simple and efficient indicator (cut-off value=48.5) for preoperative estimation of oncologic outcomes.
\end{abstract}

Key words: Colorectal cancer; Liver metastases; PNI; Prognosis

\section{Introduction}

For patients with colorectal cancer liver metastasis, hepatic resection is the main curative choice (CRLM)[1]. However, approximately $75 \%$ of patients experience tumor recurrence after the first 
liver resection[2], and only $16 \%$ of patients remain disease-free for 10 years after hepatectomy[3]. Therefore, identifying efficient prognostic factors is urgently needed to screen for high-risk subgroups and subsequently to optimize therapeutic interventions to achieve maximum therapeutic effectiveness.

Many studies have demonstrated that the progression of cancer is determined not only by tumor features but also by nutritional and immunological conditions[4-6]. The prognostic nutritional index (PNI), which is calculated according to serum albumin levels and peripheral lymphocyte numbers, reflects both the nutritional and immune status of the patient[7]. To date, some studies have demonstrated that the PNI is an indicator that could predict patients' prognosis for various malignancies, including hepatocellular carcinoma[8], renal cell carcinoma[9], pancreatic cancer[10], and gastric carcinoma[11]. However, to our knowledge, no studies have focused on the predictive role of PNI as an effective indicator of the prognosis of CRLM patients who are undergoing curative hepatic resection. In addition, the optimal cut-off value of PNI for the prediction of oncologic outcomes remains controversial. In this study, we aimed to identify the optimal PNI cut-off value and to investigate the prognostic impact of the preoperative PNI in patients with CRLM who are undergoing curative hepatic resection.

\section{Materials and Methods}

\section{Patients}

We reviewed the medical records of CRLM patients who underwent hepatic resection from September 1999 to July 2015. From the 355 cases identified, we excluded patients with palliative resection of primary tumors $(n=15)$ and palliative resection of liver metastases $(n=35)$ as well as those missing preoperative data, which would make it impossible to calculate the PNI $(n=62)$. Finally, 243 CRLM patients were included in the study. The enrolled patients met the following inclusion criteria: 1) histologically confirmed colorectal adenocarcinoma, 2) metastases to the liver, and 3) R0 resection for both the primary tumor and the liver metastases. The patient demographics, the primary and metastatic tumor characteristics, the perioperative treatment, and the follow-up results were reviewed in detail from the medical records and the follow-up system of the Sun Yat-sen University Cancer Center. All the procedures performed in studies involving human participants were in accordance with the 1964 Helsinki Declaration and its later amendments or comparable ethical standards. Institutional review board approval was also obtained from the independent ethics committee at the Sun Yat-sen University Cancer Center. Informed consent was waived in this noninterventional, observational, and retrospective study, in which the patient data were kept strictly confidential.

\section{Systemic inflammation markers}

Blood samples were collected from the patients no more than 7 days prior to the hepatic operation and analyzed for the complete blood count with differential and blood chemistry tests including albumin, tumor markers including carcinoembryonic antigen (CEA) and carbohydrate antigen 19-9 (CA199), and other laboratory tests. The PNI was calculated as $10 \times$ the serum albumin concentration $(\mathrm{g} / \mathrm{dL})+0.005 \times$ the total lymphocyte count (per $\mathrm{mm}^{3}$ ). There was no validated cut-off value for the PNI from the previous studies, so the optimal cut-off value used for the PNI in this study was calculated by the X-tile software.

\section{Follow-up}

All patients received the recommendation to return for subsequent visits every 3 months for 2 years after being discharged from the hospital, followed by semi-annual visits for an additional year. The follow-up evaluation included a routine blood test, analysis of the levels of CEA and CA19-9, abdominal ultrasonography, and chest radiograph. Chest computed tomography, abdominal/pelvic magnetic resonance imaging, and colonoscopy were performed annually. Overall survival (OS) was defined as the duration from the hepatic resection to death from any cause, while recurrence-free survival (RFS) was defined as the time interval between hepatic resection to disease recurrence or death. The follow-up period was terminated in December 2017.

\section{Statistical analysis}

Continuous variables are represented as the median (range) or the mean (standard deviation), and categorical variables are represented as percentages. The X-tile 3.6.1 software (Yale University, New Haven, CT, USA) was used to determine the optimal cut-off value of PNI, which was identified from the minimum $\mathrm{P}$ value according to the OS. Statistical analyses were performed using SPSS 20.0 software (IBM, Chicago, IL, USA) and GraphPad Prism 7 software (GraphPad Software, Inc, San Diego, CA, USA). We compared continuous variables that were normally distributed using Student's $t$ test and compared categorical variables using the chi-square $\left(x^{2}\right)$ test, Fisher's test or the nonparametric Mann-Whitney $U$ test. Survival outcomes were calculated with the Kaplan-Meier method, and the 

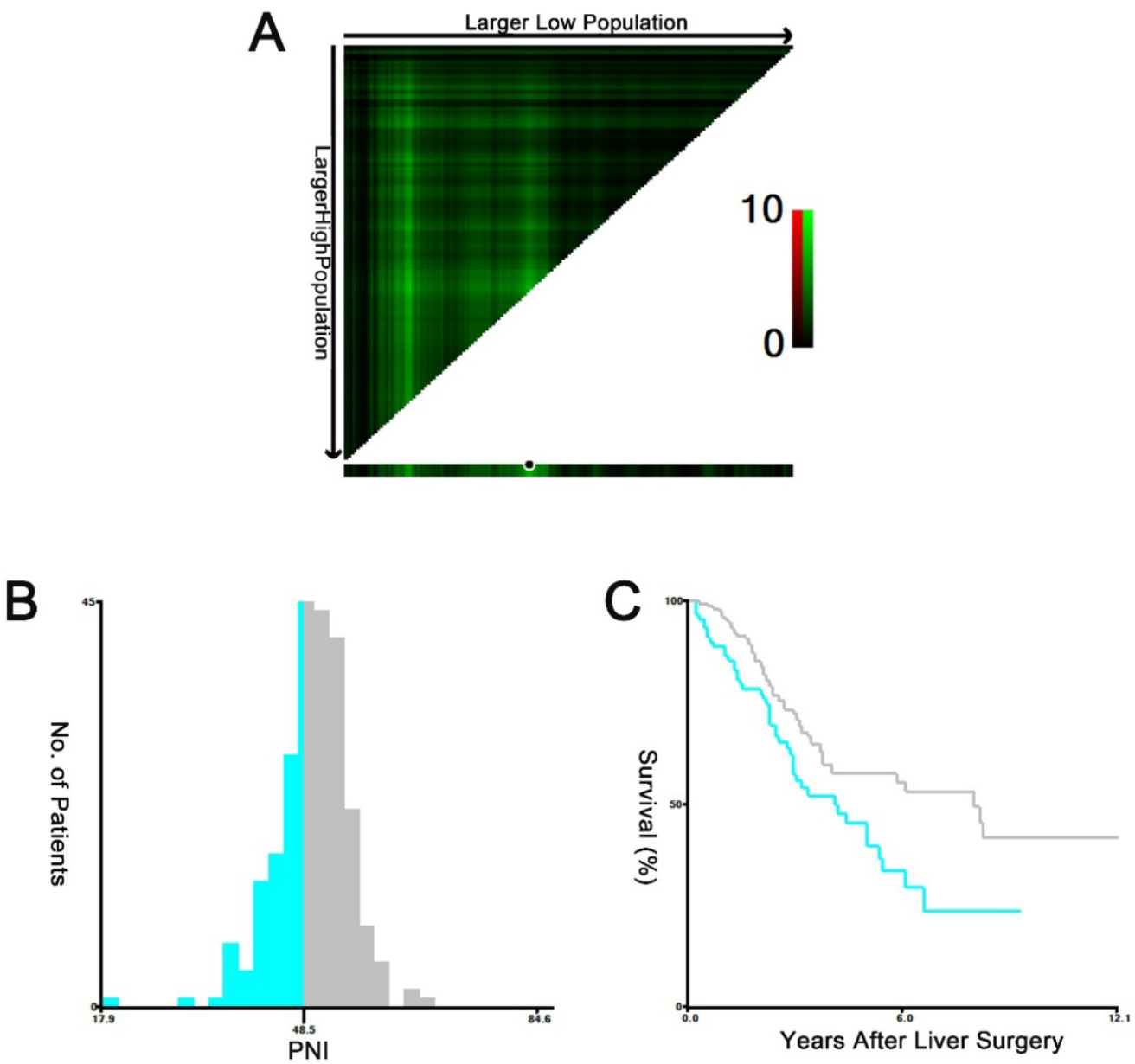

Figure 1. The $X$-tile plots of the PNI and the OS of CRLM patients who underwent curative hepatic resection. The $X$-tile plots show the $X^{2}$ values with cut-off values, generating the low- and high-PNI subgroups. A X-tile plots of PNI expression for optimal cutoff point, which is demarcated by a black circle. B The histogram of the entire cohort divided into low-PNI and high-PNI subgroups according to the optimal cut-off value of 48.5. C Kaplan-Meier plot of survival rates in groups stratified by the optimal cut-off value of the $\mathrm{PNI}$. Blue represents the low-PNI group, and gray represents the high-PNI group.

statistical significance was compared using the log-rank test. Variables that were statistically significant with a $\mathrm{P}<0.05$ in univariate Cox models were further assessed with multivariate Cox models using a forward stepwise method. The multivariate Cox proportional hazard model was used to further identify the independent prognostic factors for RFS and OS. Hazard ratios (HRs) and 95\% confidence intervals (CI) were calculated. A P value less than 0.05 was considered statistically significant.

\section{Results}

\section{Optimal cutoff value of PNI}

X-tile software was used to determine 48.5 as the optimal cut-off value for the PNI, which was identified from the minimum $P$ value according to the OS (Figure 1). In the subsequent analysis, the PNI values were divided into low-level $(\leq 48.5)$ and high-level (>48.5) groups according to the PNI before hepatic resection.

\section{Patient characteristics}

A total of 243 CRLM patients who underwent curative liver resection were analyzed in this study. The median age of the patients was 56 years (range, 25-82 years). Of the total patients, there were 151 (62.1\%) men and 92 (37.9\%) women; 154 (63.4\%) patients had colon cancer, and $89(36.6 \%)$ patients had rectal cancer. In 138 (56.8\%) patients, the primary tumors involved lymph node metastasis. Most patients $(172 / 243,70.8 \%)$ had synchronous liver metastases at the time of initial diagnosis. For the hepatic metastases, the median size of the largest tumor was $3.0 \mathrm{~cm}$ (range $0.3-12.0 \mathrm{~cm}$ ). In addition, 125 (51.4\%) patients received synchronous resection for the primary tumor and the liver metastases. With respect to chemotherapy, 78 (32.1\%) patients received neoadjuvant chemotherapy before the hepatectomy, and $184(75.7 \%)$ patients received postoperative chemotherapy. The detailed clinical and pathological characteristics of all included patients are shown in Table 1. 
Table 1. Clinicopathologic features of patients involved in this study.

\begin{tabular}{|c|c|c|}
\hline \multicolumn{2}{|l|}{ Characteristics } & \multirow{2}{*}{$\begin{array}{l}\begin{array}{l}\text { Total } \\
n=243(\%)\end{array} \\
152(62.6)\end{array}$} \\
\hline Age (years) & $\leq 60$ & \\
\hline & $>60$ & $91(37.4)$ \\
\hline \multirow[t]{2}{*}{ Sex } & Female & $92(37.9)$ \\
\hline & Male & $151(62.1)$ \\
\hline \multirow{2}{*}{ Primary tumor site } & Colon & $154(63.4)$ \\
\hline & Rectum & 89 (36.6) \\
\hline \multirow[t]{3}{*}{ Primary tumor size $(\mathrm{cm})$} & $\leq 4$ & $114(46.9)$ \\
\hline & $>4$ & $79(32.5)$ \\
\hline & Not available & $50(20.6)$ \\
\hline \multirow[t]{4}{*}{ T stage } & 1 & $2(0.8)$ \\
\hline & 2 & $18(7.4)$ \\
\hline & 3 & $139(57.2)$ \\
\hline & 4 & $84(34.6)$ \\
\hline \multirow[t]{3}{*}{$\mathrm{N}$ stage } & 0 & $105(43.2)$ \\
\hline & 1 & $80(32.9)$ \\
\hline & 2 & $58(23.9)$ \\
\hline \multirow[t]{2}{*}{ Histological grade } & Well/moderate & $208(85.6)$ \\
\hline & Poor & $35(14.4)$ \\
\hline \multirow[t]{2}{*}{ Size of liver metastases (cm) } & $\leq 2.5$ & $131(53.9)$ \\
\hline & $>2.5$ & $112(46.1)$ \\
\hline \multirow[t]{2}{*}{ Number of liver metastases } & Single & $117(48.1)$ \\
\hline & Multiple & $126(51.9)$ \\
\hline \multirow[t]{2}{*}{ Extrahepatic metastasis } & Yes & $21(8.6)$ \\
\hline & No & $222(91.4)$ \\
\hline \multirow[t]{2}{*}{ Hepatic resection timing } & Metachronous & $118(48.6)$ \\
\hline & Synchronous & $125(51.4)$ \\
\hline \multirow{4}{*}{$\begin{array}{l}\text { Chemotherapy regimen before } \\
\text { hepatic resection }\end{array}$} & Including irinotecan & $19(7.8)$ \\
\hline & Including oxaliplatin & $55(22.6)$ \\
\hline & Others & $4(1.6)$ \\
\hline & No chemotherapy & $165(67.9)$ \\
\hline \multirow{4}{*}{$\begin{array}{l}\text { Chemotherapy regimen after } \\
\text { hepatic resection }\end{array}$} & Including irinotecan & $46(18.9)$ \\
\hline & Including oxaliplatin & $125(51.4)$ \\
\hline & Others & $13(5.3)$ \\
\hline & No chemotherapy & $59(24.3)$ \\
\hline \multirow[t]{2}{*}{ RFA therapy a } & Yes & $28(11.5)$ \\
\hline & No & $215(88.5)$ \\
\hline \multirow[t]{2}{*}{ CEA level (ng/ml) b } & $\leq 5$ & $95(39.1)$ \\
\hline & $>5$ & $148(60.9)$ \\
\hline \multirow[t]{2}{*}{ CA19-9 level $(\mathrm{U} / \mathrm{ml})$ c } & $\leq 37$ & $174(71.6)$ \\
\hline & $>37$ & $69(28.4)$ \\
\hline
\end{tabular}

a Radiofrequency ablation therapy within 3 months before or after hepatic resection b Carcinoembryonic antigen before hepatic resection

c Cancer antigen 19-9 before hepatic resection

\section{Association between PNI and clinicopathological features}

The associations between the PNI status and various clinicopathological features are shown in Table 2. Among all 243 patients, there were no significant differences in the distribution of sex, tumor location or TN stage between the two groups. Additionally, patients in the two groups had similar sizes of primary tumors and liver metastases as well as similar numbers of liver metastases. Moreover, there were no significant differences in the histological grade, CEA level, or CA199 level of the patients in the low- and high-PNI groups. Older patients (age $>60$ years) and those who received synchronous hepatic resection were more frequently observed in the low-PNI group ( $\mathrm{P}=0.023$ and $\mathrm{P}=0.001)$.

Table 2. Relationships between PNI and patient characteristics

\begin{tabular}{|c|c|c|c|}
\hline \multirow[t]{2}{*}{ Characteristics } & \multicolumn{3}{|l|}{ PNI (n=243) } \\
\hline & $\begin{array}{l}\leq 48.5(\mathrm{n}=90), \\
\mathrm{n}(\%)\end{array}$ & $\begin{array}{l}>48.5(\mathrm{n}=153), \\
\mathrm{n}(\%)\end{array}$ & $\begin{array}{l}P \\
\text { value }\end{array}$ \\
\hline \multicolumn{4}{|l|}{ Age (years) } \\
\hline$\leq 60$ & $48(53.3)$ & $104(68.0)$ & 0.023 \\
\hline$>60$ & $42(46.7)$ & $49(32.0)$ & \\
\hline \multicolumn{4}{|l|}{ Sex } \\
\hline Female & $30(33.3)$ & $62(40.5)$ & 0.265 \\
\hline Male & $60(66.7)$ & $91(59.5)$ & \\
\hline \multicolumn{4}{|c|}{ Primary tumor site } \\
\hline Colon & $59(65.6)$ & $95(62.1)$ & 0.588 \\
\hline Rectum & $31(34.4)$ & $58(37.9)$ & \\
\hline \multicolumn{4}{|c|}{ Primary tumor size $(\mathrm{cm})^{a}$} \\
\hline$\leq 4$ & $40(44.4)$ & $74(48.4)$ & 0.101 \\
\hline$>4$ & $37(41.1)$ & $42(27.5)$ & \\
\hline Not available & $13(14.4)$ & $37(24.2)$ & \\
\hline \multicolumn{4}{|l|}{ T stage } \\
\hline $1-3$ & $62(68.9)$ & $97(63.4)$ & 0.385 \\
\hline 4 & $28(31.1)$ & $56(36.6)$ & \\
\hline \multicolumn{4}{|l|}{ N stage } \\
\hline 0 & $39(43.3)$ & $66(43.1)$ & 0.976 \\
\hline $1-2$ & $51(56.7)$ & $87(56.9)$ & \\
\hline \multicolumn{4}{|c|}{ Histological grade } \\
\hline Well/moderate & $75(83.3)$ & $133(86.9)$ & 0.441 \\
\hline Poor & $15(16.7)$ & $20(13.1)$ & \\
\hline \multicolumn{4}{|c|}{ Size of liver metastases (cm) } \\
\hline$\leq 2.5$ & $50(55.6)$ & $81(52.9)$ & 0.693 \\
\hline$>2.5$ & $40(44.4)$ & $72(47.1)$ & \\
\hline \multicolumn{4}{|c|}{ Number of liver metastases } \\
\hline Single & $46(51.1)$ & $71(46.4)$ & 0.478 \\
\hline Multiple & $44(48.9)$ & $82(53.6)$ & \\
\hline \multicolumn{4}{|c|}{ Extrahepatic metastasis } \\
\hline Yes & $7(7.8)$ & $14(9.2)$ & 0.713 \\
\hline No & $83(92.2)$ & $139(90.8)$ & \\
\hline \multicolumn{4}{|c|}{ Hepatic resection timing } \\
\hline Metachronous & $31(34.4)$ & $87(56.9)$ & 0.001 \\
\hline Synchronous & $59(65.6)$ & $66(43.1)$ & \\
\hline \multicolumn{4}{|c|}{$\begin{array}{l}\text { Chemotherapy regimen before } \\
\text { hepatic resection }\end{array}$} \\
\hline Yes & $32(35.6)$ & $46(30.1)$ & 0.376 \\
\hline No & $58(64.4)$ & $107(69.9)$ & \\
\hline \multicolumn{4}{|c|}{$\begin{array}{l}\text { Chemotherapy regimen after } \\
\text { hepatic resection }\end{array}$} \\
\hline Yes & $63(70.0)$ & $121(79.1)$ & 0.111 \\
\hline No & $27(30.0)$ & $32(20.9)$ & \\
\hline \multicolumn{4}{|l|}{ RFA therapy ${ }^{b}$} \\
\hline Yes & $10(11.1)$ & $18(11.8)$ & 0.878 \\
\hline No & $80(88.9)$ & $135(88.2)$ & \\
\hline \multicolumn{4}{|c|}{ CEA level $(\mathrm{ng} / \mathrm{ml}) \mathrm{c}$} \\
\hline$\leq 5$ & $40(44.4)$ & $55(35.9)$ & 0.190 \\
\hline$>5$ & $50(55.6)$ & 98 (64.1) & \\
\hline \multicolumn{4}{|c|}{ CA19-9 level $(\mathrm{U} / \mathrm{ml}) \mathrm{d}$} \\
\hline$\leq 37$ & $61(67.8)$ & $113(73.9)$ & 0.310 \\
\hline$>37$ & $29(32.2)$ & $40(26.1)$ & \\
\hline
\end{tabular}

a Data of 193 patients were available

b Radiofrequency ablation therapy within 3 months before or after hepatic resection c Carcinoembryonic antigen before hepatic resection

d Cancer antigen 19-9 before hepatic resection

\section{Association between PNI and survival}

During the follow-up period, the median OS times for the patients in the high-PNI and low-PNI groups were 95.7 months and 48.5 months, 


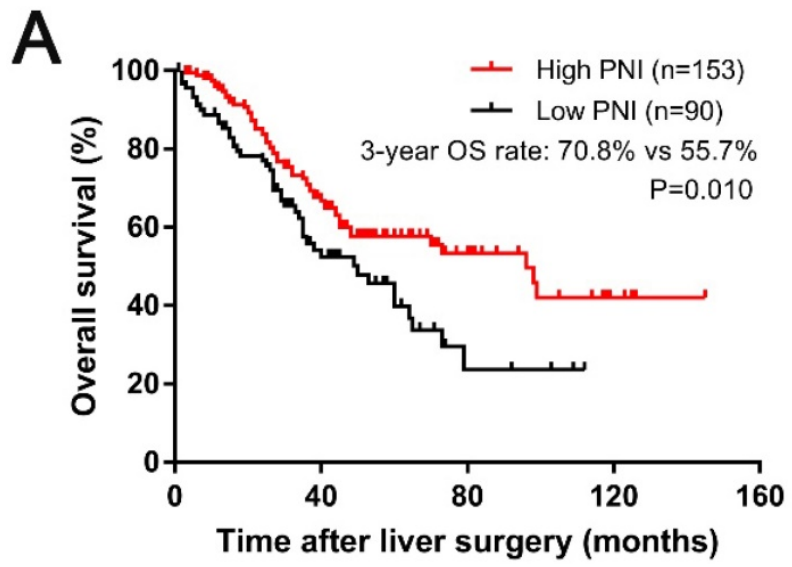

Figure 2. Kaplan-Meier curves for 3-year OS (A) and RFS (B) based on the PNI.

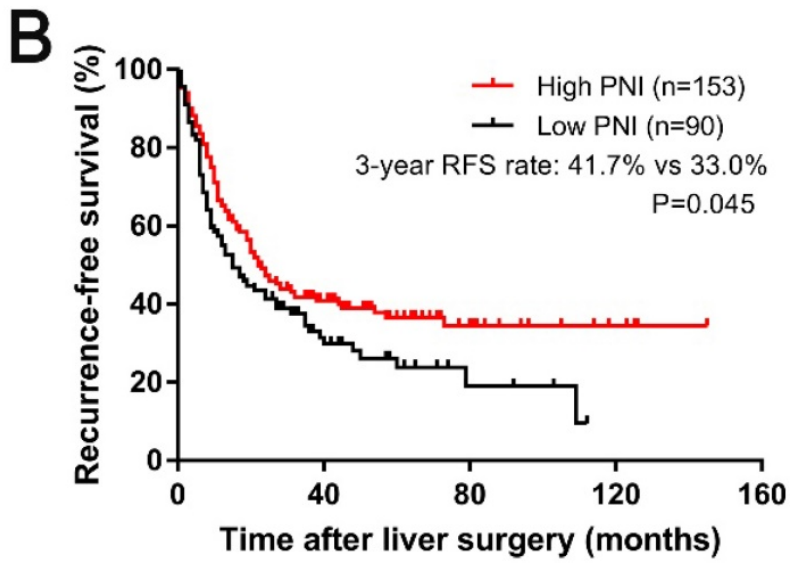

respectively. In addition, the median follow-up time after liver surgery was 36.6 months (range, 0.3-145.4 months). At the last follow-up, 107 (44.0\%) patients had died. The 1-, 3-, and 5-year OS rates were $92.9 \%$, $65.6 \%$, and $52.1 \%$, respectively. The relationships between the PNI and the survival outcomes are presented in Fig. 2. A low PNI value was associated with poor OS ( $\mathrm{P}=0.01$, shown in Figure $2 \mathrm{~A})$. The 1-, 3-, and 5 -year OS rates were $87.6 \%, 55.7 \%$, and $39.6 \%$ in the low-PNI group, respectively, and $96.0 \%, 70.8 \%$, and $57.4 \%$ in the high-PNI group, respectively. Furthermore, patients in the high-PNI group had longer RFS times than those in the low-PNI group $(\mathrm{P}=0.045$, shown in Figure 2B). In the patients from the low-PNI group, the 1-, 3-, and 5-year RFS rates were $57.3 \%, 33.0 \%$, and $26.1 \%$, respectively, compared with $66.4 \%, 41.7 \%$, and $36.5 \%$, respectively, in the high-PNI group.

The univariate analysis revealed that a high PNI value was associated with longer OS ( $\mathrm{HR}=0.606$; $95 \%$ $\mathrm{CI}=0.412-0.890 ; \mathrm{P}=0.011)$ and RFS ( $\mathrm{HR}=0.725 ; 95 \%$ $\mathrm{CI}=0.528-0.995 ; \mathrm{P}=0.046)$, as shown in Table 3 . In contrast, lymph node metastases, multiple liver metastases and undergoing radiofrequency ablation (RFA) therapy were correlated with shorter RFS and OS. Additionally, older patients and those who did not receive chemotherapy after hepatic resection had shorter OS times. Patients with larger liver metastases, higher CEA level, or chemotherapy before hepatic resection had shorter RFS. An additional Cox multivariate analysis indicated that low PNI was a nagative independent prognostic factor for OS (HR = $0.574,95 \% \mathrm{CI}=0.387-0.851, \mathrm{P}=0.006$ Table 3 ) and RFS $(\mathrm{HR}=0.650,95 \% \mathrm{CI}=0.472-0.897, \mathrm{P}=0.009)$. In addition, lymph node metastases, multiple liver metastases and RFA therapy were all independent predictors of poor OS and RFS for patients with CRLM who underwent hepatic resection. In addition, patients who received no chemotherapy after hepatic resection had worse OS, and those with higher CEA levels had worse RFS (Table 3).

\section{Discussion}

The preoperative nutritional and immunological conditions of patients have been found to be associated with both the surgical and oncologic outcomes of malignant tumors[6]. Recently, various indices containing nutritional and inflammatory parameters have been introduced to predict surgical risks and oncologic outcomes[12-14]. Among them, the PNI, a simple and useful systemic inflammation-based prognostic score, is calculated based on laboratory assessments of total lymphocyte counts and serum albumin levels and can reflect the host immunological and nutritional status[7]. Low PNI scores were first found to be predictive of a high risk of short-term postoperative complications in the gastrointestinal tract. Recently, increasing evidence has revealed that low PNI scores are also related to reduced survival in various types of malignant tumors[10, 11, 15]. However, few studies have investigated the significance of PNI as a prognostic factor for survival in CRLM patients who have undergone curative liver resection. In this retrospective study, we investigated the clinical significance of preoperative PNI scores in 243 patients with CRLM who underwent curative liver resection. The results showed that low PNI scores were associated with older age of the patient. In addition, low PNI scores were more likely to be observed in patients who underwent synchronous hepatic resection. We also demonstrated the association of preoperative PNI scores with oncologic outcomes. From the multivariate analysis, the PNI score was a negative independent predictor for both OS and RFS in CRLM patients who underwent curative liver resection. 
Table 3. Univariate and multivariate analyses using Cox proportional hazards models using factors influencing OS and RFS

\begin{tabular}{|c|c|c|c|c|c|c|c|c|}
\hline \multirow[t]{2}{*}{ Clinicaopathological features } & \multicolumn{2}{|c|}{ OS univariate analysis } & \multicolumn{2}{|c|}{ Multivariate analysis } & \multicolumn{2}{|c|}{ RFS univariate analysis } & \multicolumn{2}{|c|}{ Multivariate analysis } \\
\hline & HR $(95 \%$ CI) & P value & HR $(95 \%$ CI $)$ & $\mathrm{P}$ value & HR $(95 \%$ CI $)$ & P value & $\mathrm{HR}(95 \% \mathrm{CI})$ & P value \\
\hline \multicolumn{9}{|l|}{ PNI } \\
\hline$\leq 48.5$ vs. $>48.5$ & $0.606(0.412-0.890)$ & 0.011 & $0.574(0.387-0.851)$ & 0.006 & $0.725(0.528-0.995)$ & 0.046 & $0.650(0.472-0.897)$ & 0.009 \\
\hline \multicolumn{9}{|l|}{ Age (years) } \\
\hline$\leq 60$ vs. $>60$ & $1.468(1.002-2.150)$ & 0.049 & & & $1.074(0.780-1.477)$ & 0.662 & & \\
\hline \multicolumn{9}{|l|}{ Sex } \\
\hline Female vs. Male & $1.323(0.886-1.977)$ & 0.172 & & & $1.235(0.891-1.711)$ & 0.205 & & \\
\hline \multicolumn{9}{|l|}{ Primary tumor site } \\
\hline Colon vs. Rectum & $1.310(0.892-1.922)$ & 0.168 & & & $1.100(0.800-1.514)$ & 0.557 & & \\
\hline \multicolumn{9}{|l|}{ Primary tumor size $(\mathrm{cm})^{a}$} \\
\hline$\leq 4$ vs. $>4$ & $0.796(0.509-1.245)$ & 0.318 & & & $0.798(0.557-1.142)$ & 0.216 & & \\
\hline \multicolumn{9}{|l|}{ T stage } \\
\hline $1-3$ vs. 4 & $1.222(0.824-1.812)$ & 0.320 & & & $1.093(0.789-1.514)$ & 0.593 & & \\
\hline \multicolumn{9}{|l|}{ N stage } \\
\hline 0 vs. $1-2$ & $1.505(1.014-2.234)$ & 0.043 & $1.653(1.110-2.461)$ & 0.013 & $1.655(1.197-2.289)$ & 0.002 & $1.856(1.336-2.578)$ & $<0.001$ \\
\hline \multicolumn{9}{|l|}{ Histological grade } \\
\hline Well/moderate vs. Poor & $1.295(0.788-2.130)$ & 0.308 & & & $1.042(0.674-1.611)$ & 0.853 & & \\
\hline \multicolumn{9}{|l|}{ Size of liver metastases $(\mathrm{cm})$} \\
\hline$\leq 2.5$ vs. $>2.5$ & $1.428(0.975-2.092)$ & 0.067 & & & $1.537(1.125-2.101)$ & 0.007 & & \\
\hline \multicolumn{9}{|l|}{ Number of liver metastases } \\
\hline Single vs. Multiple & $1.834(1.238-2.718)$ & 0.002 & $1.940(1.281-2.938)$ & 0.002 & $1.823(1.325-2.508)$ & $<0.001$ & $1.750(1.249-2.452)$ & 0.001 \\
\hline \multicolumn{9}{|l|}{ Extrahepatic metastasis } \\
\hline Yes vs. No & $0.575(0.321-1.030)$ & 0.063 & & & $0.618(0.369-1.038)$ & 0.069 & & \\
\hline \multicolumn{9}{|l|}{ Liver metastasis timing } \\
\hline Metachronous vs. Synchronous & $0.733(0.500-1.073)$ & 0.110 & & & $0.762(0.558-1.041)$ & 0.088 & & \\
\hline \multicolumn{9}{|c|}{ Chemotherapy before hepatic resection } \\
\hline Yes vs. No & $0.796(0.534-1.186)$ & 0.262 & & & $0.610(0.441-0.844)$ & 0.003 & & \\
\hline \multicolumn{9}{|c|}{ Chemotherapy after hepatic resection } \\
\hline Yes vs. No & $1.709(1.118-2.614)$ & 0.013 & $1.705(1.107-2.624)$ & 0.015 & $1.162(0.809-1.668)$ & 0.417 & & \\
\hline \multicolumn{9}{|l|}{ RFA therapy ${ }^{b}$} \\
\hline Yes vs. No & $0.523(0.311-0.881)$ & 0.015 & $0.558(0.323-0.965)$ & 0.037 & $0.412(0.267-0.635)$ & $<0.001$ & $0.430(0.273-0.680)$ & $<0.001$ \\
\hline \multicolumn{9}{|l|}{ CEA level (ng/ml) c } \\
\hline$\leq 5$ vs. $>5$ & $1.249(0.840-1.856)$ & 0.272 & & & $1.453(1.046-2.019)$ & 0.026 & $1.460(1.046-2.037)$ & 0.026 \\
\hline \multicolumn{9}{|l|}{ CA19-9 level (U/ml) d } \\
\hline$\leq 37$ vs. $>37$ & $1.446(0.965-2.166)$ & 0.074 & & & $1.191(0.847-1.675)$ & 0.315 & & \\
\hline $\begin{array}{l}\text { HR hazard ratio, CI confidence inte } \\
\text { a Data of } 193 \text { patients were availabl } \\
\text { b Radiofrequency ablation therapy } \\
\text { c Carcinoembryonic antigen before } \\
\text { d Cancer antigen } 19-9 \text { before hepati }\end{array}$ & $\begin{array}{l}\text { onths before or after } \\
\text { ection }\end{array}$ & ic rese & & & & & & \\
\hline
\end{tabular}

Several nutritional and immunological parameters have been identified as being useful for the prediction of cancer patients' prognoses [16-19]. Among these, the PNI has been widely used because of its efficiency, simplicity, and convenience for clinical work. The PNI has been used to assess the preoperative condition of and to predict the surgical risk for gastrointestinal malignancy patients[7]. It is calculated based on only two factors, namely the serum albumin concentration and the lymphocyte count in the peripheral blood. The PNI was first suggested to be a nutritional index and a predictor of surgical risk, and this result was further verified by another study[8, 10]. Since then, numerous recent studies have demonstrated that a low PNI score is an independent adverse prognostic factor for short-term postoperative complications and long-term outcomes in many kinds of cancer. As is well known, the prognoses of cancer patients rely on a number of clinicopathological factors, such as TNM stage and histological type. PNI scores, however, have also been found to be closely correlated with cancer prognosis in certain types of cancer. Therefore, theoretically, PNI scores should be associated with some of those clinicopathological characteristics. In our study, we found that older patients (age $>60$ years) and those who received synchronous hepatic resection were more frequently observed in the low-PNI score group.

As a prognostic indicator, preoperative PNI has the advantage of being easily acquired because the serum albumin level and the total lymphocyte count are basic parameters that are assessed before surgery. However, an accurate cut-off value of PNI scores to use to group patients has not been determined. In a variety of malignancies, the cut-off value was determined as a wide range of 40-51[9, 20-24]. The same situation exists in colorectal cancer (CRC), for which a uniform cut-off value has not been established, with each study presenting a different value[16, 25-28]. Tokunaga et al. found that the optimal cut-off value of PNI was 45.5 using classification and regression tree analysis for OS[28]. 
Jian-hui et al. established the cut-off value as 45 according to receiver operating characteristic curve (ROC) curve analysis[25]. In our large cohort study, with the help of X-tile software using the OS of CRLM patients who underwent curative hepatic resection, we found that the optimal cut-off value for preoperative PNI scores was 48.5. PNI scores $\leq 48.5$ were defined as the low-PNI group, and scores $>48.5$ were defined as the high-PNI group. By classifying patients with this cut-off value of 48.5, significant differences in RFS and OS were observed in the present study. In clinical work, with the help of a PNI score cut-off value, we can group CRLM patients into 2 categories. Patients with high PNI scores (>48.5) may have a relatively poor survival compared to those with low PNI scores $(\leq 48.5)$. When patients with low PNI scores have two options for therapeutic regimens, they can choose the less aggressive option as the optimal one. In addition, these patients can consider undertaking a less strict follow-up schedule, considering the cost of follow-up examinations.

This study has some limitations. First, it was a retrospective study with data collected from a single institution, so potential inaccuracies may have occurred during the documentation of the medical records. However, the larger sample size provided adequate statistical power to explore the association of preoperative PNI scores with oncologic outcomes. Another limitation was the relatively short median follow-up period. However, because approximately $75 \%$ of patients experience recurrence within 2 years, the median follow-up duration of 36.6 months after hepatic resection based on clinical practice was not regarded as short. Last, it was retrospective in design. The timing of the preoperative total lymphocyte counts and the serum albumin level measurements was not always the same, which could bias the results. However, we attempted to measure both parameters within a 1-week timeframe before liver surgery.

The present study demonstrated that preoperative PNI scores with a cut-off value of 48.5 constituted a simple and efficient indicator to estimate the survival of CRLM patients who underwent curative hepatic resection. Older patients (age $>60$ years) and those who received synchronous hepatic resection were more likely to have lower preoperative PNI scores. A high preoperative PNI score is an independent prognostic factor for poor oncologic outcome including the OS and RFS of patients with CRLM undergoing hepatic resection.

\section{Abbreviations}

PNI: prognostic nutritional index; CRLM: colorectal liver metastases; RFS: regression-free survival; OS: overall survival; HR: hazard ratio; CI: confidence interval; CEA: carcinoembryonic antigen; CA199: carbohydrate antigen 19-9; RFA: radiofrequency ablation; $\mathrm{CRC}$ : colorectal cancer; ROC: receiver operating characteristic curve.

\section{Acknowledgements}

We would like to acknowledge the patients who participated in this study. We also gratefully acknowledge the assistance of the colleagues at State Key Laboratory of Oncology in South China and the Department of Colorectal Surgery in Sun Yat-sen University Cancer Center. The authenticity of the data in this article has been validated by uploading the key raw data onto the Research Data Deposit public platform (www.researchdata.org.cn), with the approval number as RDDB2018000298.

\section{Competing Interests}

The authors have declared that no competing interest exists.

\section{References}

1. Grundmann RT. Current state of surgical treatment of liver metastases from colorectal cancer. World J Gastrointest Surg. 2011; 3: 183-196.

2. D'Angelica M, Kornprat P, Gonen M, et al. Effect on outcome of recurrence patterns after hepatectomy for colorectal metastases. Ann Surg Oncol. 2011; 18: 1096-1103.

3. Tomlinson JS, Jarnagin WR, DeMatteo RP, et al. Actual 10-year survival after resection of colorectal liver metastases defines cure. J Clin Oncol. 2007; 25: 4575-4580.

4. Mantovani A, Allavena P, Sica A, Balkwill F. Cancer-related inflammation. Nature. 2008; 454: 436-444.

5. Balkwill F, Mantovani A. Inflammation and cancer: back to Virchow? Lancet. 2001; 357: 539-545.

6. Schwegler I, von Holzen A, Gutzwiller JP, et al. Nutritional risk is a clinical predictor of postoperative mortality and morbidity in surgery for colorectal cancer. Br J Surg. 2010; 97: 92-97.

7. Onodera T, Goseki N, Kosaki G. Prognostic nutritional index in gastrointestinal surgery of malnourished cancer patients. Nihon Geka Gakkai Zasshi. 1984; 85: 1001-1005

8. Chan AW, Chan SL, Wong GL, et al. Prognostic Nutritional Index (PNI) Predicts Tumor Recurrence of Very Early/Early Stage Hepatocellular Carcinoma After Surgical Resection. Ann Surg Oncol. 2015; 22: 4138-4148.

9. Jeon HG, Choi DK, Sung HH, et al. Preoperative Prognostic Nutritional Index is a Significant Predictor of Survival in Renal Cell Carcinoma Patients Undergoing Nephrectomy. Ann Surg Oncol. 2016; 23: 321-327.

10. Kanda M, Fujii T, Kodera $Y$, et al. Nutritional predictors of postoperative outcome in pancreatic cancer. Br J Surg. 2011; 98: 268-274.

11. Migita K, Takayama T, Saeki K, et al. The prognostic nutritional index predicts long-term outcomes of gastric cancer patients independent of tumor stage. Ann Surg Oncol. 2013; 20: 2647-2654.

12. Hirashima $\mathrm{K}$, Watanabe $\mathrm{M}$, Shigaki $\mathrm{H}$, et al. Prognostic significance of the modified Glasgow prognostic score in elderly patients with gastric cancer. J Gastroenterol. 2014; 49: 1040-1046.

13. Kwag SJ, Kim JG, Kang WK, et al. The nutritional risk is a independent factor for postoperative morbidity in surgery for colorectal cancer. Ann Surg Treat Res. 2014; 86: 206-211.

14. Watanabe M, Iwatsuki M, Iwagami $\mathrm{S}$, et al. Prognostic nutritional index predicts outcomes of gastrectomy in the elderly. World J Surg. 2012; 36: 1632-1639.

15. Pinato DJ, North BV, Sharma R. A novel, externally validated inflammation-based prognostic algorithm in hepatocellular carcinoma: the prognostic nutritional index (PNI). Br J Cancer. 2012; 106: 1439-1445.

16. Nozoe T, Ninomiya M, Maeda T, et al. Prognostic nutritional index: a tool to predict the biological aggressiveness of gastric carcinoma. Surg Today. 2010; 40: $440-443$.

17. Asher V, Lee J, Innamaa A, Bali A. Preoperative platelet lymphocyte ratio as an independent prognostic marker in ovarian cancer. Clin Transl Oncol. 2011; 13: 499-503.

18. Chua W, Charles KA, Baracos VE, Clarke SJ. Neutrophil/lymphocyte ratio predicts chemotherapy outcomes in patients with advanced colorectal cancer. Br J Cancer. 2011; 104: 1288-1295. 
19. Li QQ, Lu ZH, Yang L, et al. Neutrophil count and the inflammation-based glasgow prognostic score predict survival in patients with advanced gastric cancer receiving first-line chemotherapy. Asian Pac J Cancer Prev. 2014; 15: 945-950.

20. Jeon HG, Choi DK, Sung HH, et al. Preoperative Prognostic Nutritional Index is a Significant Predictor of Survival in Renal Cell Carcinoma Patients Undergoing Nephrectomy. Ann Surg Oncol. 2016; 23: 321-327.

21. Sakurai K, Ohira M, Tamura T, et al. Predictive Potential of Preoperative Nutritional Status in Long-Term Outcome Projections for Patients with Gastric Cancer. Ann Surg Oncol. 2016; 23: 525-533.

22. Shoji F, Morodomi Y, Akamine T, et al. Predictive impact for postoperative recurrence using the preoperative prognostic nutritional index in pathological stage I non-small cell lung cancer. Lung Cancer. 2016; 98: 15-21.

23. Sun $\mathrm{K}, \mathrm{Chen} \mathrm{S}, \mathrm{Xu} \mathrm{J}$, et al. The prognostic significance of the prognostic nutritional index in cancer: a systematic review and meta-analysis. J Cancer Res Clin Oncol. 2014; 140: 1537-1549.

24. Yang Z, Zhang B, Hou L, et al. Pre-operative prognostic nutritional index predicts the outcomes for triple-negative breast cancer. Tumour Biol. 2014; 35: 12165-12171.

25. Jian-Hui C, Iskandar EA, Cai S, et al. Significance of Onodera's prognostic nutritional index in patients with colorectal cancer: a large cohort study in a single Chinese institution. Tumour Biol. 2016; 37: 3277-3283.

26. Maeda K, Shibutani M, Otani H, et al. Low nutritional prognostic index correlates with poor survival in patients with stage IV colorectal cancer following palliative resection of the primary tumor. World J Surg. 2014; 38: 1217-1222.

27. Mohri Y, Inoue $\mathrm{Y}$, Tanaka $\mathrm{K}$, et al. Prognostic nutritional index predicts postoperative outcome in colorectal cancer. World J Surg. 2013; 37: 2688-2692.

28. Tokunaga R, Sakamoto $Y$, Nakagawa S, et al. Prognostic Nutritional Index Predicts Severe Complications, Recurrence, and Poor Prognosis in Patients with Colorectal Cancer Undergoing Primary Tumor Resection. Dis Colon Rectum. 2015; 58: 1048-1057. 